\section{Rethink the classical view of cerebrospinal fluid production}

\author{
Margaux Roques (D), Amaury De Barros and Fabrice Bonneville
}

In their brilliant review, Joanna Wardlaw and colleagues describe those well-known but poorly understood CNS features, the perivascular spaces (Wardlaw, J. M. et al. Perivascular spaces in the brain: anatomy, physiology, and pathology. Nat. Rev. Neurol. 16, 137-153 $\left.(2020)^{1}\right)$. Questions persist on the role of these spaces in interstitial fluid-cerebrospinal fluid (ISF-CSF) metabolite clearance, such as drainage pathways, the role of aquaporin 4 in water transport, and the effects of sleep on their function. In their discussion, the authors accurately indicate that the occurrence of CSF drainage via arachnoid granulations in humans has been questioned. We believe that their statement that CSF is "produced continuously in the choroid plexus" should be examined too.

The traditional 'bulk flow' theory of active production of CSF in the choroid plexus and its passive absorption via arachnoid granulations - historically presented as the 'third circulation' - mostly originates from a single experiment by Dandy published in 1919 that has not been reproduced since. He performed a unilateral choroid plexectomy and obstruction of the foramen of Monro on one dog, which led to the collapse of the plexectomized ventricle and dilation of the ventricle containing a choroid plexus ${ }^{2}$. By contrast, in 1969, Milhorat et al. ${ }^{3}$ obstructed the ventricular system of 76 monkeys, which caused hydrocephalus independently of choroid plexectomy, suggesting a role for extra-choroidal sites in CSF production.

The function of the choroid plexus has frequently been investigated in in vitro and ex vivo experiments, the conditions of which could differ from physiological conditions. However, MRI can be used to explore CSF dynamics in vivo. Use of dynamic oxygenenhanced MRI has demonstrated that inhaled oxygen predominantly diffuses into the sulcal CSF rather than the ventricular $\mathrm{CSF}^{4}$. This observation could be explained by the abundance of pial arterioles relative to intraventricular vessels and the direct crossing of intra-arterial molecules through the blood-CSF barrier to form CSF. In addition, use of several imaging techniques in which CSF motion is directly visualized has demonstrated no motion around the choroid plexus, which contradicts the notion that choroid plexus arterial pulsation is the driving force for CSF pulsatile motion 5 . These in vivo findings support a new model, in which CSF is produced by water filtration across capillary walls throughout the CNS and is subjected to a combination of multidirectional motions, where hydrodynamic and osmotic changes play a crucial role 6 .

Another line of evidence that suggests that the choroid plexus is not the only site of CSF production is the failure of plexectomy to treat paediatric hydrocephalus. This approach, which was used for decades, has largely been replaced by shunt or endoscopic ventriculostomy owing to its lack of efficacy in all but exceptional cases of CP hyperplasia ${ }^{7}$. In addition, the combination of choroid plexus cauterization with ventriculostomy does not improve outcomes for children with post-infectious hydrocephalus ${ }^{8}$. Inflammation has recently been proved to play a prominent role in acquired hydrocephalus, but further experiments are required for greater clarity ${ }^{9}$.

In summary, the oversimplified bulk flow theory is commonly presented as fact in textbooks and medical schools but should be presented as one of several models, along with its limitations and flaws. Examination of the entire ISF-CSF pathway from production to clearance is essential for improving treatment of conditions that involve altered CSF dynamics, such as obstructive hydrocephalus, normal pressure hydrocephalus and even intracranial hypotension, by taking into consideration not only the symptoms but, above all, the responsible mechanisms.

There is a reply to this letter by Wardlaw et al. Nat. Rev. Neurol. https://doi.org/10.1038/ s41582-021-00539-z (2021).

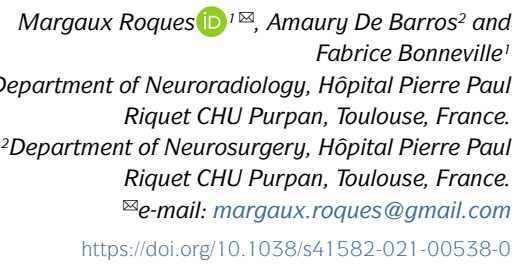

1. Wardlaw, J. M. et al. Perivascular spaces in the brain anatomy, physiology and pathology. Nat. Rev. Neurol. 16, 137-153 (2020)

2. Dandy, W. E. Experimental hydrocephalus. Ann. Surg 70, 129-142 (1919)

3. Milhorat, T. H., Hammock, M. K., Fenstermacher, J. D. Rall, D. P. \& Levin, V. A. Cerebrospinal fluid production by the choroid plexus and brain. Science 173 330-332 (1971)

4. Mehemed, T. M. et al. Dynamic oxygen-enhanced MRI of cerebrospinal fluid. PLOS ONE 9, e 100723 (2014).

5. Matsumae, M. et al. Changing the currently held concept of cerebrospinal fluid dynamics based on shared findings of cerebrospinal fluid motion in the cranial cavity using various types of magnetic resonance imaging techniques. Neurol. Med. Chir. 59, 133-146 (2019).

6. Orešković, D., Radoš, M. \& Klarica, M. New concepts of cerebrospinal fluid physiology and development of hydrocephalus. Pediatr. Neurosurg. 52, 417-425 (2017).

7. Limbrick, D. D., Baird, L. C., Klimo, P., Riva-Cambrin, J. \& Flannery, A. M. Pediatric hydrocephalus: systematic literature review and evidence-based guidelines. Part 4: Cerebrospinal fluid shunt or endoscopic third ventriculostomy for the treatment of hydrocephalus in children. J. Neurosurg. Pediatr. 14 30-34 (2014).

8. Kulkarni, A. V. et al. Endoscopic treatment versus shunting for infant hydrocephalus in Uganda. N. Engl. J. Med. 377, 2456-2464 (2017).

9. Karimy, J K et al Inflammation in acquired hydrocephalus: pathogenic mechanisms and therapeutic targets. Nat. Rev. Neurol. 16, 285-296 (2020).

\title{
Reply to: Rethink the classical view of cerebrospinal fluid production
}

\section{Joanna M. Wardlaw (D), Helene Benveniste, Maiken Nedergaard(D), Berislav V. Zlokovic, Serge Charpak, Kenneth J. Smith (1) and Sandra E. Black(D)}

We thank Roques and colleagues for their comments on our Review (Wardlaw, J. M. et al. Perivascular spaces in the brain: anatomy, physiology, and pathology. Nat. Rev. Neurol. 16, 137-153 $\left.(2020)^{1}\right)$, in which they correctly point out some textbook dogma that needs to be re-evaluated (Roques, M., De Barros, A. \& Bonneville, F. Rethink the classical view of cerebrospinal fluid production. Nat. Rev. Neurol. https://doi.org/10.1038/ s41582-021-00538-0 (2021 $\left.)^{2}\right)$. We agree that the questions of how much cerebrospinal fluid (CSF) is produced and from which structures remain unanswered. In our Review, we noted that there are several potential sources of CSF, including the choroid plexus, but information about the relative contributions of each source is unfortunately limited.

In our Review ${ }^{1}$, we focused on perivascular spaces as seen in vivo on human brain 